\title{
A Monetary Incentive DTN Routing For Wireless Sensor Networks
}

\section{Zhi Lin}

Beijing Normal University https://orcid.org/0000-0002-1837-9982

\section{Xia Wang ( $\nabla$ wangxia@bjut.edu.cn )}

Beijing University of Technology

\author{
Rongfang Bie \\ Beijing Normal University \\ Hongwei Shi \\ Beijing Normal University
}

\section{Research Article}

Keywords: KKT conditions, DTN routing, nonlinear programming

Posted Date: December 20th, 2021

DOI: https://doi.org/10.21203/rs.3.rs-1100643/v1

License: (9) This work is licensed under a Creative Commons Attribution 4.0 International License. Read Full License 


\title{
A Monetary Incentive DTN Routing for Wireless Sensor Networks
}

\author{
Zhi Lin ${ }^{1}$, Xia Wang ${ }^{2 *}$, Rongfang $\mathrm{Bie}^{3}$ and Hongwei Shi ${ }^{1}$
}

\author{
${ }^{*}$ Correspondence: \\ wangxia@bjut.edu.cn \\ ${ }^{2}$ School of Statistics and Data \\ Science, Faculty of Science, \\ Beijing University of Technology, \\ Beijing, CN \\ Full list of author information is \\ available at the end of the article
}

\begin{abstract}
The DTN (Delay/Interrupt Tolerant Network) protocol that relies on nodes to handle network interruptions is one of the important components of the wireless sensor network (WSN) routing protocol. However, due to resource consumption, nodes may be unable to unconditionally relay data. To address this issue, several incentive mechanisms have recently been proposed to encourage node participation. However, the existing solutions either do not fully consider the sender's budget or do not consider the relay cost limitation, which violated the practical incentive mechanism requirements of the DTN protocol.

In this paper, we focus on developing a new incentive mechanism for DTN routing that specifically address the challenges brought up by budget and relay cost limitation. Our core idea is to define the payoff functions of the sender and the next hops, then optimize the strategies under the constraints of the sender's budget and the relay's cost. Our experimental results demonstrate that the maximized social welfare for all participants can be realized under these constraints.
\end{abstract}

Keywords: KKT conditions; DTN routing; nonlinear programming

\section{Introduction}

Wireless Sensor Networks (WSNs) are self-organized networks consisting of spatially distributed sensors monitoring and responding to physical and environmental conditions. WSN can be self-configurable, self-optimized, self-healing, and self-scheduling. However, the mobility of sensor nodes may cause frequent disconnections between the source and destination. Thus, there is a network partition problem in WSN. To address this issue, the Delay/Interrupt Tolerant Network (DTN) protocol, created for interplanetary communication to handle long delays, was later expanded to support WSNs dealing with disruptions [1].

The key design of the DTN protocol is to transfer data by store-carry-forward operation during opportunistic connections $[2,3]$. Such mechanisms are based on a common assumption that all nodes are ready to transmit data for others. However, this is not always true, because the resources of mobile nodes (battery life, storage space, bandwidth, and computing capability) are limited, and nodes would not forward bundles for others unconditionally.

Without enough participation in routing, DTN data transmission may fail. In order to solve this problem, an effective solution is to use monetary incentives to encourage nodes to participate in routing. Sugiyama et al. [4] presented a method where mobile operators offload cellular traffic through DTN. However, the reward for each DTN subscriber is fixed. It could not be adjusted according to the packet 
amount that the operator transmits to the subscriber. Huang et al. [5] proposed a solution considering the data amount assigned to one particular node and the payment for that node. But it does not take the budget limitation or the cost burden into account, which is a fundamental issue in reality.

In this work, we propose a new incentive mechanism for DTN routing that specifically address the challenges brought up by budget and relay cost limitation. Specifically, a node that is going to send the bundle in DTN is a requestor, or a sender in DTN Bundle Protocol [6], while the node that is ready to be the next hop is the worker, or a receiver in DTN Bundle Protocol [6]. Compared with existing solutions, our proposed design has two advantages: 1) the sender pays to the relays according to the transmitted data amount; 2) the sender's budget limit and the upper bound of a worker's cost are considered. Considering two different conditions, we implement two distinct algorithms that have different optimization focus: 1) optimization under the sender's budget limit (OSBL); 2) optimization under the relays' cost upper bound (ORCU). It is worth to note that the relay's cost grows rapidly when the data amount rises. Thus, if the relay's cost grows more slowly, it may possess a greater transmission capacity. When the sender's budget is limited, the simulation of OSBL shows that if the relay has a bigger capacity, it can be assigned more data and then paid more. If the relays' capacities are big enough, the optimized result is decided by the sender's budget. On the other hand, when the relays' costs are upper bounded, the simulation of ORCU presents that if the relay has a bigger capacity, the optimal solution is less likely affected by its cost constraint.

The remaining part of the paper is organized as follows: the payoff functions of the sender and the relays are defined in Section 3. Section 4 and 5 describe the solution of optimal strategy with the sender's budget limit (OSBL) and that of the relay's cost upper bound (ORCU), respectively. The simulation results for both OSBL and ORCU cases are demonstrated in Section 6. Section 7 presents the related work in DTN routing. A conclusion of this paper is in Section 8.

\section{Methods}

Routing in a DTN of space flight is expensive, and more participants are needed in a DTN in data relay, we were challenged by a question how the routing would be if the sender needs to pay for data transmission balancing budgets.

The problem could be interpreted as to choose a relay node and calculate the payment and the data size. Fortunately, DTNs route based on Contact Graph Routing, in other words the task of choosing a relay node is turned into maintaining the information of Contact Plan. It's independent from data transmission. So we can focus on the monetary issue which is to find the optimal values of equations with inequalities constraints about budget or cost. It's a nonlinear programming problem, and the mathematical tool to solve it is to state KKT (Karush-Kuhn-Tucker) conditions.

\section{Problem Statement and Payoff Functions}

\subsection{An Introduction of DTN Architecture}

In a DTN architecture, Bundle Protocol (BP) endpoints are functionally analogous to socket layer as showed in Fig. 1 [9, 10]. BP is over LTP (Licklider Transmission 
Protocol) [7] which is analogous to TCP (Transmission Control Protocol). And a system of dynamic routing for LTP is specified as Contact Graph Routing [8].

Contact Graph Routing can be intuitively understood as each BP node has accurate "contact plan" information like Contacts in one's phone, recording the information of other nodes, including time available, data rate and the distance [8].

Based on the contact plan, whenever sending a bundle, the node computes a route based on Proximate Nodes list which is a list of neighbor nodes [8]. For now, there is no fee information in contact plan messages, and a protocol for distributing contact plan information to the BP agents are not defined. This article is about monetary incentive for neighbor nodes. It could be implemented by defining a new type of contact plan message, or be defined in the protocol for maintaining contact plan information, or be used in preparing the contact plan information.

\subsection{Payoff Functions}

Facing many acceptable hops, the sender would select workers to maximize its benefit. There are multiple transactions simultaneously in DTN, in each transaction there are one requestor and $n$ workers $(n \leq 1)$. Hence the problem can be focused on one transaction. During one transaction, the requestor and the worker will agree on one reward price. In the following part, we define the payoff functions for each player.

The payoff function of the requestor is defined as below:

$$
R_{R}=\sum_{i=1}^{n} m_{i}\left(w_{i}-p_{i}\right)
$$

where $w_{i}$ represents the price of data per unit transmitted by worker $i$. The transmission quality provided by this worker and the amount of $w_{i}$ relies on the urgency and imperativeness of the data. Besides, $p_{i}$ means the payment per data unit for worker $i . m_{i}$ is the data amount assigned to worker $i$.

The payoff function of worker $i$ is described as $R_{w i}=m_{i} p_{i}-C_{i}$, in which $C_{i}$ is the cost of transmitting data of worker $i$, and it is the function of task amount (namely data amount here). We employ the quadratic equation in this work to formulate $C_{i}$, which is a classic cost function in economic research [13]. That is, $C_{i}=\alpha_{i} m_{i}^{2}+\beta_{i} m_{i}+\gamma_{i}$.

Thus, the payoff function of worker $i$ can be presented by the following equation, that is,

$$
R_{w i}=-\alpha_{i} m_{i}^{2}+\left(p_{i}-\beta_{i}\right) m_{i}-\gamma_{i} .
$$

In practice, variables $\alpha_{i}, \beta_{i}, \gamma_{i}$ are positive and we can obtain the maximum value by derivation since the first-order derivative of $R_{w i}$ is zero, and the second-order derivative of $R_{w i}$ is $-2 \alpha_{i}<0$.

Let's optimize the payoff functions in two scenarios: 1) Optimization with the sender's budget limit (OSBL); 2) Optimization with the relays' cost upper bound $(O R C U)$. Since the two conditions are from quite different angles, they are introduced separately in two sections. 


\section{Optimization with the Sender's Budget Limit (OSBL)}

When the requestor's budget is limited, it can be mathematically defined as an inequality $\sum_{i=1}^{n} m_{i} p_{i} \leq P$. Our task is to find the values of $m_{i}$ and $p_{i}$ to reach the maximal payoff for the requestor and the workers under this constraint.

Observing the requestor sets the price $p_{i}$, the best response of worker $i$ is to maximize the profit. The condition for a maximum is that the FOC(first-order condition) with respect to $m_{i}$ is equal to zero. That is,

$$
\frac{\partial R_{w i}}{\partial m_{i}}=0 \Rightarrow m_{i}=\frac{p_{i}-\beta_{i}}{2 \alpha_{i}} .
$$

Replace $m_{i}$ by $\frac{p_{i}-\beta_{i}}{2 \alpha_{i}}$ in (1), we have

$$
R_{R}=\sum_{i=1}^{n} \frac{p_{i}-\beta_{i}}{2 \alpha_{i}}\left(w_{i}-p_{i}\right)
$$

Hence, to optimize the requestor's payoff is to find the maximum of (4), or to minimize the following equation:

$$
-R_{R}=-\sum_{i=1}^{n} \frac{p_{i}-\beta_{i}}{2 \alpha_{i}}\left(w_{i}-p_{i}\right) .
$$

And there is a constraint $\sum_{i=1}^{n} m_{i} p_{i}-P \leq 0$, where $m_{i}, p_{i} \geq 0$. This is a nonlinear programming problem with one inequality, which can be addressed via KKT (Karush-Kuhn-Tucker) conditions [14-17]. To be specific, the Lagrange function $L$ can be derived as $[15,16]$ :

$$
\begin{aligned}
& L\left(m_{1}, m_{2}, \cdots, \lambda\right)=-R_{R}+\lambda\left(\sum_{i=1}^{n} m_{i} p_{i}-P\right) \\
& =-\sum_{i=1}^{n} m_{i}\left(w_{i}-p_{i}\right)+\lambda\left(\sum_{i=1}^{n} m_{i} p_{i}-P\right) \\
& =\sum_{i=1}^{n} \frac{p_{i}^{2}-\left(\beta_{i}+w_{i}\right) p_{i}+\beta_{i} w_{i}}{2 \alpha_{i}}+\lambda\left(\sum_{i=1}^{n} \frac{p_{i}^{2}-p_{i} \beta_{i}}{2 \alpha_{i}}-P\right) .
\end{aligned}
$$

Here the set of KKT conditions which should be satisfied are the following [18]:

(Optimality)

$$
\frac{\partial L}{\partial p_{i}}=0
$$

(Feasibility)

$$
\sum_{i=1}^{n} m_{i} p_{i}-P \leq 0
$$

(Complementary Slackness) [19]

$$
\lambda\left(\sum_{i=1}^{n} m_{i} p_{i}-P\right)=0
$$


(Nonnegativity)

$$
\lambda \geq 0
$$

Subsequently, we deal with the above problem step by step. To begin with, from the optimality condition (7), we have

$$
\frac{\partial L}{\partial p_{i}}=0 \Rightarrow p_{i}=\frac{\beta_{i}}{2}+\frac{w_{i}}{2(1+\lambda)}
$$

and the complementary slackness condition (9) shows us that two cases exist:

Case I: for the inactive case, where $\lambda=0$, we have $\sum_{i=1}^{n} m_{i} p_{i}-P<0$;

Case II: for the active case, in which $\lambda>0$, we can obtain $\sum_{i=1}^{n} m_{i} p_{i}-P=0$.

In Case I, based on $\lambda=0$ and $\sum_{i=1}^{n} m_{i} p_{i}-P<0$, we have the following derivations:

$$
\begin{aligned}
& \lambda=0 \Rightarrow p_{i}^{*}=\frac{w_{i}+\beta_{i}}{2}, m_{i}^{*}=\frac{p_{i}-\beta_{i}}{2 \alpha_{i}}=\frac{w_{i}-\beta_{i}}{4 \alpha_{i}}, \\
& L^{*}=-\sum_{i=1}^{n} m_{i}^{*}\left(w_{i}-p_{i}^{*}\right)=-\sum_{i=1}^{n} \frac{\left(w_{i}-\beta_{i}\right)^{2}}{8 \alpha_{i}}, \\
& R_{R}^{*}=-L^{*}=\sum_{i=1}^{n} \frac{\left(w_{i}-\beta_{i}\right)^{2}}{8 \alpha_{i}}, \\
& R_{w i}^{*}=\frac{1}{16 \alpha_{i}}\left(w_{i}-\beta_{i}\right)^{2}-\gamma_{i},
\end{aligned}
$$

where $L^{*}$ is the minimum of Lagrange function $L$ and $R_{R}^{*}$ is the maximal value of the requestor's payoff function. As for $R_{w i}^{*}$, it shows the maximal value of worker $i$ 's payoff function.

In Case II, according to $\lambda>0$ and $\sum_{i=1}^{n} m_{i} p_{i}-P=0$, combining (11), let's set $x=\frac{1}{1+\lambda}$ where $\lambda>0$, so $x<1$ can be met. Hence, we replace $x<1$ as the constraint in Case II, based on which, the following equations can be calculated subsequently,

$$
\begin{aligned}
& p_{i}^{*}=\frac{\beta_{i}}{2}+\frac{w_{i}}{2(1+\lambda)}=\frac{1}{2}\left(w_{i} x+\beta_{i}\right), \\
& m_{i}^{*}=\frac{p_{i}-\beta_{i}}{2 \alpha_{i}}=\frac{1}{4 \alpha_{i}}\left(w_{i} x-\beta_{i}\right), \\
& \sum_{i=1}^{n} m_{i}^{*} p_{i}^{*}=P \Rightarrow \sum_{i=1}^{n} \frac{1}{8 \alpha_{i}}\left(w_{i}^{2} x^{2}-\beta_{i}^{2}\right)=P \Rightarrow \\
& x=\sqrt{\frac{P+\sum_{i=1}^{n} \beta_{i}^{2} /\left(8 \alpha_{i}\right)}{\sum_{i=1}^{n} w_{i}^{2} /\left(8 \alpha_{i}\right)}} .
\end{aligned}
$$


Next, we substitute $m_{i}, p_{i}, x$ in $L$ :

$$
\begin{aligned}
& L^{*}=-\sum_{i=1}^{n} m_{i}^{*}\left(w_{i}-p_{i}^{*}\right)=-\sum_{i=1}^{n} m_{i}^{*} w_{i}+P \\
& =-\sum_{i=1}^{n} \frac{1}{4 \alpha_{i}}\left(w_{i} \sqrt{\frac{P+\sum_{i=1}^{n} \beta_{i}^{2} /\left(8 \alpha_{i}\right)}{\sum_{i=1}^{n} w_{i}^{2} /\left(8 \alpha_{i}\right)}}-\beta_{i}\right) w_{i}+P, \\
& R_{R}^{*}=-L^{*} \\
& =\sum_{i=1}^{n} \frac{1}{4 \alpha_{i}}\left(w_{i} \sqrt{\frac{P+\sum_{i=1}^{n} \beta_{i}^{2} /\left(8 \alpha_{i}\right)}{\sum_{i=1}^{n} w_{i}^{2} /\left(8 \alpha_{i}\right)}}-\beta_{i}\right) w_{i}-P, \\
& R_{w i}^{*}=\frac{1}{16 \alpha_{i}}\left(w_{i} x-\beta_{i}\right)^{2}-\gamma_{i} \\
& =\frac{1}{16 \alpha_{i}}\left(w_{i} \sqrt{\frac{P+\sum_{i=1}^{n} \beta_{i}^{2} /\left(8 \alpha_{i}\right)}{\sum_{i=1}^{n} w_{i}^{2} /\left(8 \alpha_{i}\right)}}-\beta_{i}\right)^{2}-\gamma_{i} .
\end{aligned}
$$

From the above analysis, we now acquire two alternative values, but only one is the global optimal solution, namely the KKT point. And which one is the solution depends on the numbers of the variables.

\section{Optimization with the Relays' Costs Upper Bounded (ORCU)}

If the relays' costs are upper bounded, we can conclude that worker $i$ 's cost $\alpha_{i} m_{i}^{2}+$ $\beta_{i} m_{i}+\gamma_{i}$ is no more than $S_{i}$. In this case, we need to answer a question: what's the win-win strategy for the requestor to assign certain amounts of data to a worker with a specific price?

As mentioned in the above section, (3) and (4) are still valid in this scenario. However, since each worker has its cost limit, a new nonlinear programming problem with multiple inequalities as constraints is proposed in this part. Formally, we put it in a standardized way as follows.

To minimize:

$$
-R_{R}=-\sum_{i=1}^{n} \frac{p_{i}-\beta_{i}}{2 \alpha_{i}}\left(w_{i}-p_{i}\right),
$$

\section{Subject to:}

$$
\begin{array}{r}
\alpha_{1} m_{1}^{2}+\beta_{1} m_{1}+\gamma_{1}-S_{1} \leq 0, \\
\alpha_{1} m_{2}^{2}+\beta_{2} m_{2}+\gamma_{2}-S_{2} \leq 0, \\
\ldots \\
\alpha_{n} m_{n}^{2}+\beta_{n} m_{n}+\gamma_{n}-S_{n} \leq 0, \\
m_{i}, w_{i} \geq, 0 \\
1 \leq i \leq n .
\end{array}
$$


Hence, the Lagrange function $L$ can be described as

$$
\begin{aligned}
& L\left(m_{1}, m_{2}, \cdots, \lambda_{1}, \lambda_{2}, \cdots\right) \\
& =-R_{R}+\sum_{i=1}^{n} \lambda_{i}\left(\alpha_{i} m_{i}^{2}+\beta_{i} m_{i}+\gamma_{i}-S_{i}\right) \\
& =-\sum_{i=1}^{n} \frac{p_{i}-\beta_{i}}{2 \alpha_{i}}\left(w_{i}-p_{i}\right) \\
& +\sum_{i=1}^{n} \lambda_{i}\left[\frac{\left(p_{i}-\beta_{i}\right)^{2}}{4 \alpha_{i}}+\frac{\beta_{i}\left(p_{i}-\beta_{i}\right)}{2 \alpha_{i}}+\gamma_{i}-S_{i}\right],
\end{aligned}
$$

and the set of KKT conditions are presented as

(Optimality)

$$
\forall i, \frac{\partial L}{\partial p_{i}}=0,
$$

(Feasibility)

$$
\forall i, \alpha_{i} m_{i}^{2}+\beta_{i} m_{i}+\gamma_{i}-S_{i} \leq 0,
$$

(Complementary Slackness)

$$
\forall i, \lambda_{i}\left(\alpha_{i} m_{i}^{2}+\beta_{i} m_{i}+\gamma_{i}-S_{i}\right)=0
$$

(Nonnegativity)

$$
\forall i, \lambda_{i} \geq 0
$$

For concreteness, let's choose $i=1$ first, and generalize it to $n$ workers later.

(Optimality)

$$
\frac{\partial L}{\partial p_{1}}=0 \Rightarrow p_{1}=\frac{\beta_{1}+w_{1}}{2+\lambda_{1}},
$$

(Feasibility)

$$
\alpha_{1} m_{1}^{2}+\beta_{1} m_{1}+\gamma_{1}-S_{1} \leq 0,
$$

(Complementary Slackness)

$$
\lambda_{1}\left(\alpha_{1} m_{1}^{2}+\beta_{1} m_{1}+\gamma_{1}-S_{1}\right)=0,
$$

(Nonnegativity)

$$
\lambda_{1} \geq 0 .
$$


Likewise, the complementary slackness condition (29) tells us the following two cases hold:

Case I: when $\lambda_{1}=0$, we have $\alpha_{1} m_{1}^{2}+\beta_{1} m_{1}+\gamma_{1}-S_{1}<0$;

Case II: if $\lambda_{1}>0$, we can derive $\alpha_{1} m_{1}^{2}+\beta_{1} m_{1}+\gamma_{1}-S_{1}=0$.

For Case I, when $\lambda_{1}=0$ and $\alpha_{1} m_{1}^{2}+\beta_{1} m_{1}+\gamma_{1}-S_{1}<0$, we have

$$
p_{1}^{*}=\frac{w_{1}+\beta_{1}}{2}, m_{1}^{*}=\frac{w_{1}-\beta_{1}}{4 \alpha_{1}} .
$$

For Case II, when $\lambda_{1}>0$ and $\alpha_{1} m_{1}^{2}+\beta_{1} m_{1}+\gamma_{1}-S_{1}=0$, in the case of $\alpha_{1}>0$, $\beta_{1}^{2}-4 \alpha_{1}\left(\gamma_{1}-S_{1}\right) \geq 0$, we can obtain

$$
m_{1}=\frac{-\beta_{1} \pm \sqrt{\beta_{1}^{2}-4 \alpha_{1}\left(\gamma_{1}-S_{1}\right)}}{2 \alpha_{1}},
$$

since $m_{1}>0$, we conclude that

$$
\begin{aligned}
& m_{1}^{*}=\frac{-\beta_{1}+\sqrt{\beta_{1}^{2}-4 \alpha_{1}\left(\gamma_{1}-S_{1}\right)}}{2 \alpha_{1}}, \\
& p_{1}^{*}=\sqrt{\beta_{1}^{2}-4 \alpha_{1}\left(\gamma_{1}-S_{1}\right)}, \\
& \lambda_{1}=\frac{\beta_{1}+w_{1}}{\sqrt{\beta_{1}^{2}-4 \alpha_{1}\left(\gamma_{1}-S_{1}\right)}}-2 .
\end{aligned}
$$

As for $n$ workers, there are also solutions in two cases. The number of combinations of all the workers could be $2^{n}$ and the global optimal solution depends on numbers, where

$$
\begin{aligned}
& R_{R}^{*}=\sum_{i=1}^{n} m_{i}^{*}\left(w_{i}-p_{i}^{*}\right), \\
& R_{w i}^{*}=m_{i}^{*} p_{i}^{*}-\alpha_{i} m_{i}^{* 2}-\beta_{i} m_{i}^{*}-\gamma_{i} .
\end{aligned}
$$

\section{Results and Discussion}

This section describes the simulation results of the optimal strategy under the sender's budget limit (OSBL) and the relays' cost upper bound (ORCU), respectively.

\subsection{Simulations of OSBL}

We begin by laying out the results of the OSBL scenario. Suppose there are 3 workers, i.e., $n=3$ and budget $P=1000$. To find multiple scenarios, we list the cases when Case I is the KKT point and Case II is not, or vice versa (see Supplementary Materials for more details).

The variables $\alpha_{i}, \beta_{i}, \gamma_{i}$, and $w_{i}$ are presented in Table 1. Additionally, we also state $m_{i}, p_{i}, m_{i} * p_{i}, R_{w i}, R_{R}$, and auxiliary parameter $x$ for Case II. Conclusions can be drawn if we have done some adjustments: 1) the range of parameter suggests that in the case of it is out of range, at least one of them is negative; (2) when the limitation has been violated, the desirable KKT point is determined to be the result in the other case. The domain for each variable roughly are: $\alpha_{i}(0.1,1), \beta_{i}$ 
$[0,22], \gamma_{i}(10,50), w_{i}(20,30)$. Here in this paper, we present an example to illustrate the result due to the page limitation and other parameter settings show the same trend. To be specific, the limitation in Case I can be met when $\alpha_{i}=0.905$ while the constraint in Case II $x<1$ is violated. Thus, the KKT point comes to be the solution in Case I (see Excel file "KKT points simulation details20200205.xls" sheet "sender's budget-alpha-case-1"). On the other hand, the result changes when $\alpha_{i}=0.11$ that the KKT point goes to Case II (see the Excel file sheet "sender's budget-alpha-case-2").

Given the parameter settings in Table 1, Table 2 demonstrates its corresponding solutions. Conclusions can be drawn that: when $\alpha_{1}=0.905$, the final solution is: $m_{1}=5.80, m_{2}=9.50$, and $m_{3}=36.91$. Besides, the maximum payoff of the requestor is 557.20 and the utilities of the workers are $0.46,20.13,172.02$, respectively, with a total data amount of 52.21. Moreover, in the case of $\alpha_{1}$ has been tuned to a much smaller value as 0.1 , the solutions become $m_{1}=42.63, m_{2}=6.00$, and $m_{3}=24.11$. Besides, the maximum payoff of the requestor is 964.07 and the utilities of the workers are $144.69,1.76$, and 101.62 correspondingly, with the total data amount 77.00 .

Conclusively, the above results can be interpreted in the following ways:

(1) The payoff of the requestor may rise dramatically $(557.20 \rightarrow 964.07)$ when the cost of the worker, i.e., $\alpha_{i}$ is smaller. Besides, the payoff of worker obtains an improvement $(0.46 \rightarrow 144.69)$, making itself more powerful in the competition compared with other workers. However, the amount of total data transmitted increases accordingly $(52.21 \rightarrow 77.00)$;

(2) The maximal value occurs with a higher probability at the edge of the budget constraint when the cost of the workers is less sensitive to the task amount (the smaller the $\alpha_{i}$ is). That is to say, the higher the capacity the worker has, the less barrier the requestor is limited, but rely more on its budget;

(3) In the optimized solution, no matter how the data amounts assigned to each worker vary apparently, the prices are almost the same.

Figs. 2 and 3 demonstrate how the KKT point varies under different parameter settings. Without loss of generality, we choose $\alpha_{1}$ as an example, which varies from 0.11 to 1 while other parameters keep fixed.

The revenues of the requestor and that of the workers in Case I and Case II are shown in Figs. 2 and 3, respectively. From which we can see the critical point of $\alpha_{1}$. Specifically, for any value higher than it, the constraint $\sum_{i=1}^{3} m_{i} p_{i}<1000$ can be satisfied. As for those $\alpha_{1}$ less than it, the constraint $x<1$ can be satisfied. For clarity, the corresponding revenues of the requestor are highlighted by bold lines.

Fig. 4 demonstrates the revenues of the requestor and that of the workers in both cases, indicating that a unique critical value determines which case the KKT point is. Besides, the maximum payoff in both cases matches almost perfectly.

Exploring the workers' revenues further in Fig. 4, we obtain Fig. 5, which can be interpreted as follows.

(1) In the case of a smaller $\alpha_{1}$ and the constraint $x<1$ is satisfied, the KKT point may fall in the range of Case II. Besides, with the increase of $\alpha_{1}$, worker 1's payoff drops while worker 2's payoff increases. Such a trend implies that $\alpha_{1}$ affects competition to a great extent. That is to say, if the cost of worker 1 rises more 
rapidly when the task amount is larger, it wins less revenue. On the contrary, the revenues of worker 2 and worker 3 rise up;

(2) When the condition $\sum_{i=1}^{3} m_{i} p_{i}<1000$ is satisfied, the KKT point will fall in the area of Case I. Moreover, worker 1's payoff decreases as $\alpha_{1}$ increases, but those of worker 2 and worker 3 keep fixed, indicating that if worker 1 is too weak in controlling cost, its achievement doesn't affect others' revenues.

Now we turn to check how the KKT point varies as $\beta_{1}$ changes. Keep other parameters untouched in Table 1, increasing $\beta_{1}$ to its upper bound, i.e., 17.

Figs. 6 and 7 depict the revenues changing with $\beta_{1}$ in Case I and II, respectively. The former figure is under the constraint of $\sum_{i=1}^{3} m_{i} p_{i}<1000$ which is divided by 2. And the parameter $x$ of the latter one is limited by $x<1$ and the results have been multiplied by 300 times for better display.

Regardless of the change of $\beta_{1}$, the KKT points remain in the area of Case I. A similar situation happens in Case II until $\beta_{1}>15.64$. But at the very end of the curve, the KKT points transfer to Case I. It is reasonable to conclude that $\alpha_{1}$ weighs more than $\beta_{1}$ in deciding which case the KKT point is. Additionally, $\gamma_{i}$ does not influence the payoff of the requestor. And the change of $\gamma_{1}$ is only associated with worker 1's payoff and does not impact the other two workers' revenues. It doesn't change the conclusion that which case is the KKT point.

\subsection{Simulations of ORCU}

We set the parameters in Table 3 (see the Excel file sheet "The worker's cost"), the solutions are shown in Tables 4 and 5 .

The feasibilities are $\alpha_{1} m_{1}^{2}+\beta_{1} m_{1}+\gamma_{1}-S_{1}<0$ and $\lambda_{1}>0$ in Case I and II. We can see that the solutions of worker 1 and worker 3 which are underlined lie in Case 1 and the solution of worker 2 is in Case II. Besides, the utilities of the requestor and the workers are:

$$
\begin{aligned}
& R_{R}^{*}=34.51+62.27+192.91=229.69 \\
& R_{w 1}^{*}=7.25 \\
& R_{w 2}^{*}=10.13 \\
& R_{w 3}^{*}=66.46
\end{aligned}
$$

which can be explained in this way:

(1) The bigger the cost threshold is, the more likely the solution lies in Case I, resulting in the optimal solution is free from the constraint to a greater extent $\left(\lambda_{1}=0\right.$, the constraint is ignored);

(2) If the worker's cost hardly relies on the task amount ( $\alpha_{i}$ is small), the solution is unlikely limited by the constraint, that is, the solution stays mostly in Case I.

Fig. 8 shows the constraint lines of worker 1 both in Case I and Case II, marked as dashed lines. In detail, the constraint in Case I is $\alpha_{1} m_{1}^{2}+\beta_{1} m_{1}+\gamma_{1}-S_{1}<0$, so the valid area lies on the right side of the intersection point, redrawn as a line. The constraint in Case II is $\lambda_{1}>0$ (the value is multiplied by 10 for better representation in the graph), so the valid area lies on the left side of the intersection point, also redrawn as a line.

Other workers are similar. And the final solution is defined by the valid solutions of all the workers. 


\section{Related Work}

There are two types of incentive algorithms in DTN routing, one is reputation-based [22-27], and the other is price-based [5, 20, 21, 28-30].

$\mathrm{Hu}$ et al. [22] proposed a solution to set TTL based on budget, which can be tuned due to the network traffic. Bonnefon et al. [23] introduced surveys on what people prefer when Autonomous Vehicles (AVs) face conflicts of saving pedestrians or passengers. Hilbe et al. [24] analyzed strategies that people with better self-control and coordination could win more in a large group. [25] showed in the experiment that a group identity among players enhanced cooperations. Ning et al. [26] put forward an approach where the receiver provides credit when it meets interesting content from the sender. Chen et al. [27] said the credit value should be associated with QoS. And the method is to send the first packets with higher benefits and drop those with lower ones.

[5] applied the crowdsourcing model in DTN routing originally and a nonlinear programming framework with equality limitations was proposed thereafter. At present, researchers have devoted much attention to DTN routing algorithms. Dini Duca [21] designed a mechanism where each node locally records the reputation of forwarding nodes, based on which the next hops can be chosen. Lu et al. [28] encouraged users to forward others' bundles to improve their package delivery. Uddin et al. [29] devised to rank nodes based on their contribution, deciding message priority accordingly. Ning et al. [30] proposed two types of incentives based on whether a node is within a social community.

Inspired by Huang et al. [5], we go on to solve a nonlinear programming problem with inequality constraints utilizing KKT conditions.

This paper is a revised and extended version of a conference paper entitled "A Mechanism Design Solution for DTN Routing", presented at International Conference on Identification, Information and Knowledge in the Internet of Things (IIKI) 2016, Oct. 20, Beijing. The differences are as below:

- A new constraint that the relays costs are upper bounded;

- A revision in solving the problem under the first constraint that the sender's budget is limited, especially different data in simulation.

\section{Conclusion}

In this paper, we introduce a monetary incentive solution in DTN data transmission service. In both situations, either the sender's budget is limited or the relays' costs are upper-bounded, the optimized solution for all the participants exist. They can be mathematically solved and be verified by simulation. The capacity of a relay decides the task amount assigned. The more competitive a relay is, the more tasks and payment it gets. But the price per task for different relays stays roughly the same. For further work, we plan to deploy the algorithm in a real DTN application.

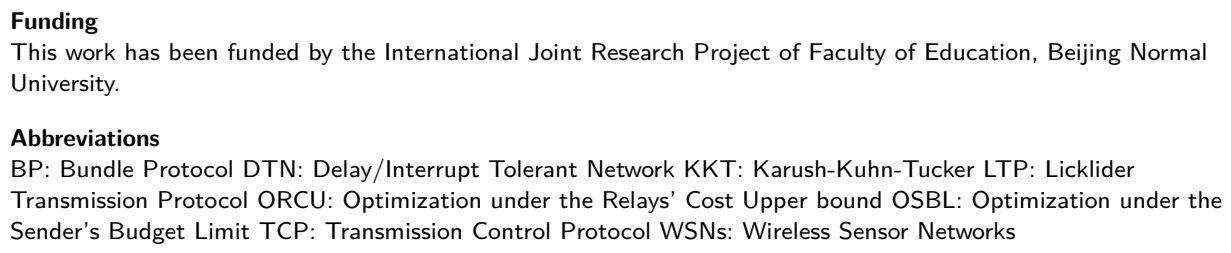




\section{Availability of data and materials}

The Excel file "KKT points simulation details20200205.xls" is available online at shorturl.at/ajCD3.

\section{Competing interests}

The authors declare that they have no competing interests.

Authors' contributions

Zhi Lin is responsible for writing the manuscript. Rongfang Bie teaches the algorithm. Hongwei Shi participated in drafting the manuscript.

\section{Acknowledgements}

Prof. Shengling Wang directed us in research of DTN domain. And She also guided us in defining the problem and solving it. Mr. Yu Guo helped to polish the manuscript.

Author details

${ }^{1}$ School of Artificial Intelligence, Beijing Normal University, Beijing, CN. ${ }^{2}$ School of Statistics and Data Science, Faculty of Science, Beijing University of Technology, Beijing, CN. ${ }^{3}$ Center of Information \& Network Technology, Beijing Normal University, Beijing, CN.

\section{References}

References

1. Fall, K. and Farrell, S., 2008. DTN: an architectural retrospective. IEEE Journal on Selected areas in communications, 26(5), pp.828-836.

2. Fall, K., Hong, W. and Madden, S., 2003. Custody transfer for reliable delivery in delay tolerant networks. IRB-TR-03-030, July.

3. Symington, S., Durst, R.C. and Scott, K., 2007, January. Custodial Multicast in Delay Tolerant Networks. In CCNC (pp. 207-211).

4. Sugiyama, K., Kubo, T., Tagami, A. and Parekh, A., 2013, December. Incentive mechanism for DTN-based message delivery services. In 2013 IEEE Global Communications Conference (GLOBECOM) (pp. 3108-3113). IEEE.

5. Huang, J., Hu, Q., Bi, J. and Li, Z., 2016, August. Stackelberg game based incentive mechanism for data transmission in mobile opportunistic networks. In International Conference on Wireless Algorithms, Systems, and Applications (pp. 377-388). Springer, Cham

6. Bundle Protocol Version 7, IRTF, 2020, https://tools.ietf.org/html/draft-ietf-dtn-bpbis-25

7. M. Ramadas, S. Burleigh, S. Farrell, RFC5326: Licklider Transmission Protocol - Specification, September 2008, IETF, https://datatracker.ietf.org/doc/html/rfc5326

8. S. Burleigh, Contact Graph Routing, draft-burleigh-dtnrg-cgr-01, July 8, 2010, https://datatracker.ietf.org/doc/html/draft-burleigh-dtnrg-cgr

9. Scott Burleigh, ION IMPLEMENTATION OF THE DTN ARCHITECTURE, 2020, https://www.nasa.gov/sites/default/files/atoms/files/1.1_lecture_-_intro_to_dtn_implentation_ of_the_dtn_architecture.pdf

10. Scott Burleigh, ION IMPLEMENTATION OF THE DTN ARCHITECTURE, 2020, California Institute of Technology, https://www.nasa.gov/sites/default/files/atoms/files/1.2_lecture_-_intro_to_ion_ implentation_of_the_dtn_architecture.pdf

11. Zhao, W., Ammar, M. and Zegura, E., 2005, August. Multicasting in delay tolerant networks: semantic models and routing algorithms. In Proceedings of the 2005 ACM SIGCOMM workshop on Delay-tolerant networking (pp. 268-275).

12. Guo, S., Derakhshani, M., Falaki, M.H., Ismail, U., Luk, R., Oliver, E.A., Rahman, S.U., Seth, A., Zaharia, M.A. and Keshav, S., 2011. Design and implementation of the KioskNet system. Computer Networks, 55(1), pp.264-281.

13. D D Tewari, 1996. "Principles of Microeconomics", page 136, 1996, https://goo.gl/RQceJv

14. Kuhn, H.W. and Tucker, A.W., 2014. Nonlinear programming. In Traces and emergence of nonlinear programming (pp. 247-258). Birkhäuser, Basel.

15. Strang, G., 1988. Linear algebra and its applications, Thomson Learning. Inc., London.

16. Josephine Sullivan, 2012. "Lagrange Multipliers and the Karush-Kuhn-Tucker conditions", March 20, 2012, http://www.csc.kth.se/utbildning/kth/kurser/DD3364/Lectures/KKT.pdf

17. Stanley B. Gershwin, 2010. "KKT Examples", Massachusetts Institute of Technology, http://ocw.mit.edu/courses/mechanical-engineering/ 2-854-introduction-to-manufacturing-systems-fall-2010/lecture-notes/MIT2_854F10_kkt_ex.pdf

18. Debjani Chakraborty, 2014. "Mod-01 Lec-24 Nonlinear programming KKT conditions", IIT Kharagpur, 2014.6.10, https://www. youtube.com/watch?v=_Lt3zNdnEOk

19. Hugo, "Karush-Kuhn-Tucker (KKT) conditions", http://www. onmyphd.com/?p=kkt. karush. kuhn. tucker 20. Moreira, W. and Mendes, P., 2010. Survey on opportunistic routing for delay/disruption tolerant networks.

21. Dini, G. and Duca, A.L., 2012. Towards a reputation-based routing protocol to contrast blackholes in a delay tolerant network. Ad Hoc Networks, 10(7), pp.1167-1178.

22. Hu, Y., Feng, M., Bhuyan, L.N. and Kalogeraki, V., 2009, April. Budget-based self-optimized incentive search in unstructured P2P networks. In IEEE INFOCOM 2009 (pp. 352-360). IEEE.

23. Bonnefon, J.F., Shariff, A. and Rahwan, I., 2016. The social dilemma of autonomous vehicles. Science, 352(6293), pp.1573-1576.

24. Hilbe, C., Wu, B., Traulsen, A. and Nowak, M.A., 2014. Cooperation and control in multiplayer social dilemmas. Proceedings of the National Academy of Sciences, 111(46), pp.16425-16430. 
25. Dawes, R.M., Van De Kragt, A.J. and Orbell, J.M., 1988. Not me or thee but we: The importance of group identity in eliciting cooperation in dilemma situations: Experimental manipulations. Acta Psychologica, 68(1-3) pp.83-97.

26. Ning, T., Yang, Z., Xie, X. and Wu, H., 2011, June. Incentive-aware data dissemination in delay-tolerant mobile networks. In 2011 8th Annual IEEE Communications Society Conference on Sensor, Mesh and Ad Hoc Communications and Networks (pp. 539-547). IEEE.

27. Chen, K., Shen, H. and Yan, L., 2014. Multicent: A multifunctional incentive scheme adaptive to diverse performance objectives for DTN routing. IEEE Transactions on Parallel and Distributed Systems, 26(6), pp.1643-1653.

28. Lu, R., Lin, X., Zhu, H., Shen, X. and Preiss, B., 2010. Pi: A practical incentive protocol for delay tolerant networks. IEEE transactions on wireless communications, 9(4), pp.1483-1493.

29. Uddin, M.Y.S., Godfrey, B. and Abdelzaher, T., 2010, October. RELICS: In-network realization of incentives to combat selfishness in DTN. In The 18th IEEE International Conference on Network Protocols (pp. 203-212). IEEE.

30. Ning, Z., Liu, L., Xia, F., Jedari, B., Lee, I. and Zhang, W., 2016. CAIS: A copy adjustable incentive scheme in community-based socially aware networking. IEEE Transactions on Vehicular Technology, 66(4), pp.3406-3419. 
Figures

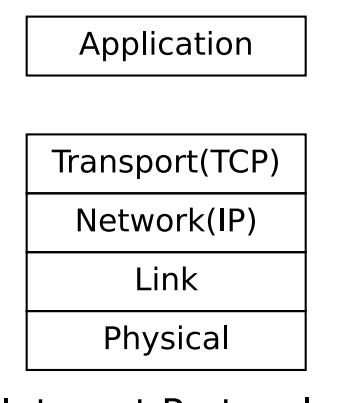

\begin{tabular}{|c|}
\hline Application \\
\hline BP \\
\hline LTP \\
\hline Network \\
\hline Link \\
\hline Physical \\
\hline
\end{tabular}

Internet Protocols

DTN Protocols

Figure 1 DTN Protocols

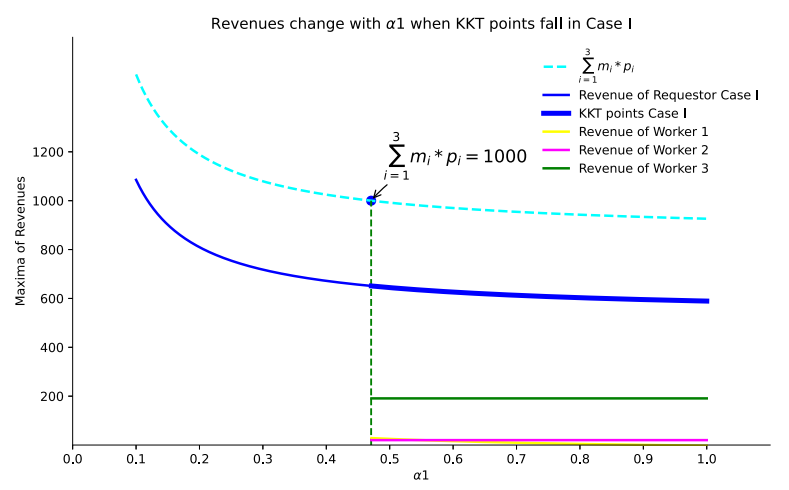

Figure 2 Revenues change with $\alpha_{1}$ in Case I 


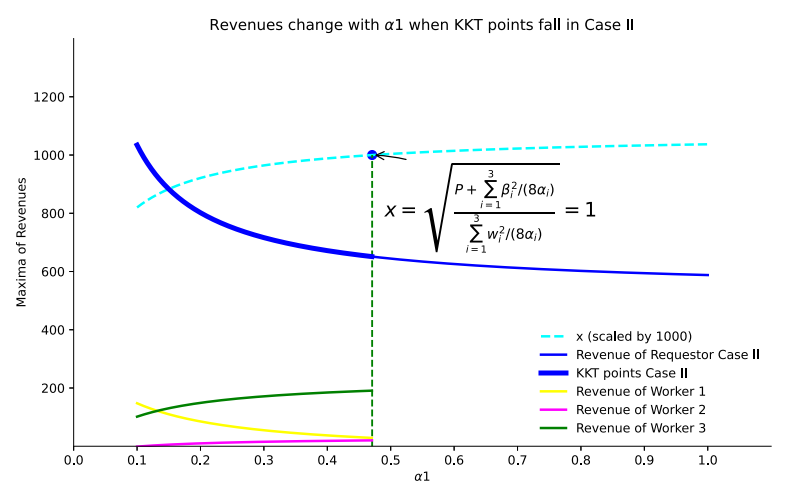

Figure 3 Revenues change with $\alpha_{1}$ in Case II

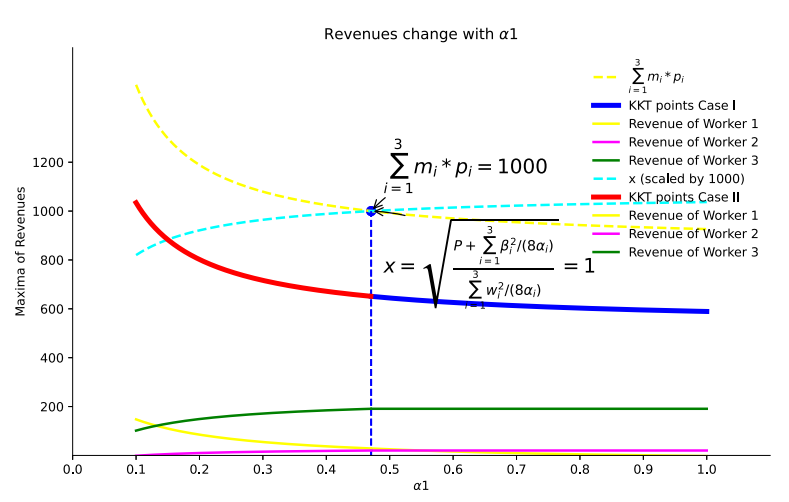

Figure 4 Revenues change with $\alpha_{1}$

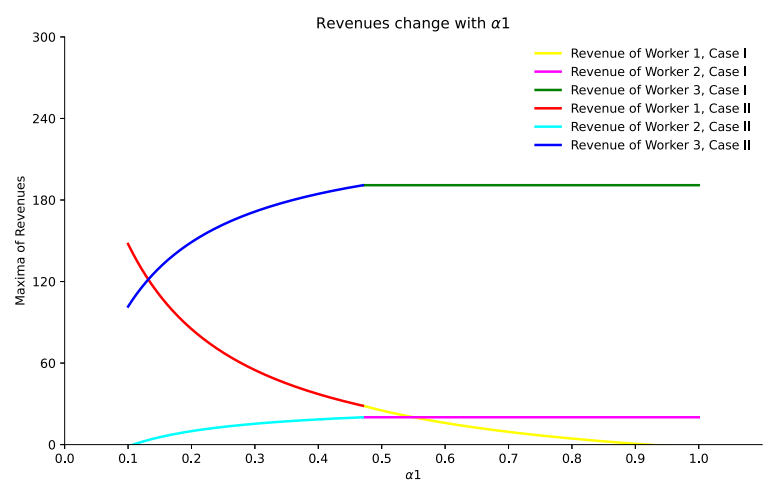

Figure 5 Workers' revenues change with $\alpha_{1}$ 


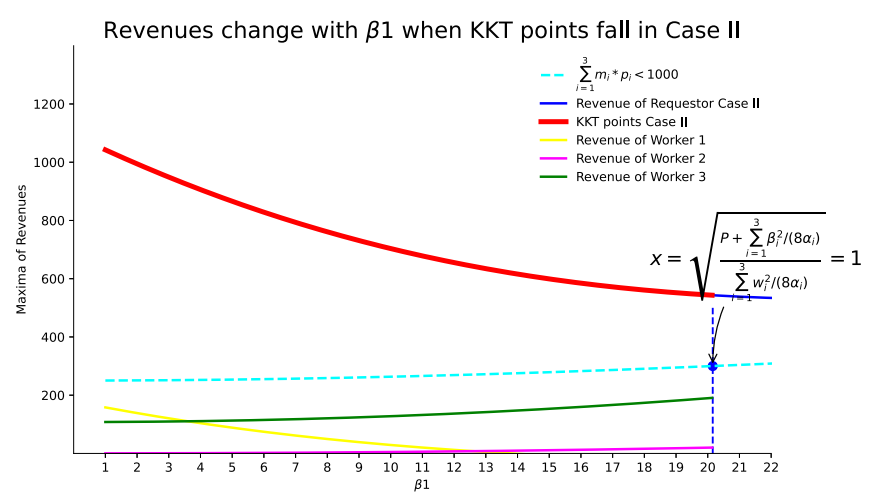

Figure 7 Revenues change with $\beta_{1}$ in Case II

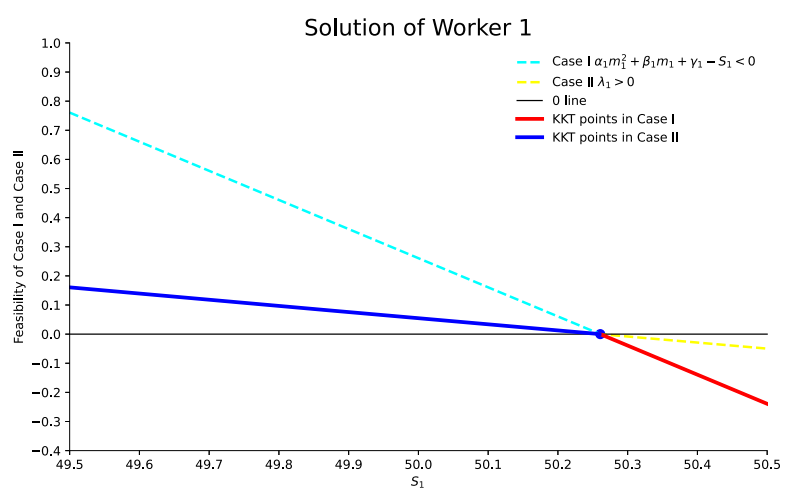

Figure 8 The valid solution of Worker 1 
Tables

Table 1 The parameters setting

\begin{tabular}{cccccc}
\hline$i$ & $\alpha_{i}$ Case I & $\alpha_{i}$ Case II & $\beta_{i}$ & $\gamma_{i}$ & $w_{i}$ \\
\hline 1 & 0.905 & 0.11 & 2 & 30 & 23 \\
2 & 0.5 & 0.5 & 10 & 25 & 29 \\
3 & 0.149 & 0.149 & 6 & 31 & 28 \\
Domains & $(0.1,1)$ & $(0.1,1)$ & {$[0,17]$} & $(10,50)$ & $(20,30)$ \\
\hline
\end{tabular}

Table 2 Solutions

\begin{tabular}{ccccccccc}
\hline$i$ & $\alpha_{i}$ Case I & $m_{i}$ Case I & $p_{i}$ Case I & $R_{w i}$ Case I & $\alpha_{i}$ Case II & $m_{i}$ Case II & $p_{i}$ Case II & $R_{w i}$ Case II \\
\hline 1 & 0.905 & 5.80 & 12.5 & 0.46 & 0.11 & 39.85 & 10.77 & 144.69 \\
2 & 0.5 & 9.50 & 19.5 & 20.13 & 0.5 & 7.32 & 17.32 & 1.76 \\
3 & 0.149 & 36.91 & 17 & 172.02 & 0.149 & 29.83 & 14.89 & 101.62 \\
$R_{R}$ & & & & 557.20 & & & & \\
\multicolumn{2}{r}{} & & & & & & & \\
\hline
\end{tabular}

Table 3 The parameters setting for ORCU

\begin{tabular}{cccccc}
\hline$i$ & $\alpha_{i}$ & $\beta_{i}$ & $\gamma_{i}$ & $w_{i}$ & $S_{i}$ \\
\hline 1 & 0.815 & 1 & 10 & 29 & 51 \\
2 & 0.58 & 9 & 20 & 25 & 115 \\
3 & 0.127 & 7 & 30 & 21 & 320 \\
Domains & $(0.147,1)$ & {$[0,17]$} & $(10,50)$ & $(20,30)$ & $S_{i}>\beta_{i}$ \\
\hline
\end{tabular}

Table 4 Solutions in CASE I

\begin{tabular}{ccccc}
\hline$m_{i}$ & $p_{i}$ & $m_{i}\left(w_{i}-p_{i}\right)$ & $R_{w i}$ & Feasibility \\
\hline 4.60 & 12.5 & $\underline{34.51}$ & $\underline{7.25}$ & $\frac{-0.74}{2.09}$ \\
7.33 & 17.5 & $\underline{62.28}$ & $\underline{11.14}$ & $\underline{66.46}$ \\
27.56 & 14 & $\underline{-0.63}$ \\
\hline
\end{tabular}

Table 5 Solutions in CASE II

\begin{tabular}{ccccc}
\hline$m_{i}$ & $p_{i}$ & $m_{i}\left(w_{i}-p_{i}\right)$ & $R_{w i}$ & $\lambda_{i}$ \\
\hline 4.66 & 12.60 & 34.50 & 7.70 & -0.015 \\
7.21 & 17.36 & $\underline{62.27}$ & $\underline{10.13}$ & $\underline{0.016}$ \\
27.60 & 14.01 & 192.91 & 6.77 & $\begin{array}{c}-0.002 \\
>0\end{array}$ \\
\hline
\end{tabular}




\section{Supplementary Files}

This is a list of supplementary files associated with this preprint. Click to download.

- KKTpointssimulationdetails20200205.xlsx 